from 46 public STD clinics in the 4 provinces. Specimens that tested positive for $C$. trachomatis by polymerase chain reaction were genotyped for the infecting C. trachomatis strain by amplifying and sequencing the genovar-specific ompA gene, which encodes the chlamydial major outer membrane protein. Nine C. trachomatis genovars were identified from 129 specimens; they consisted of the $\mathrm{F}$ (25.6\%, $\mathrm{n}=33), \mathrm{E}(17.1 \%, \mathrm{n}=22), \mathrm{J}(16.3 \%, \mathrm{n}=21), \mathrm{D}(15.5 \%$, $\mathrm{n}=20), \mathrm{G}(11.6 \%, \mathrm{n}=15), \mathrm{K}(5.4 \%, \mathrm{n}=7), \mathrm{H}(3.9 \%, \mathrm{n}=5), \mathrm{I}(1.6 \%$, $\mathrm{n}=2)$, and $\mathrm{B}(0.8 \%, \mathrm{n}=1)$ genovars. Nine genovars were found in specimens from Guangxi province, 6 were found in specimens from each of Guangdong and Hainan provinces, but only 5 were found in specimens from Jiangsu province. Significant differences were observed in C. trachomatis genovar distributions between different provinces: G/Ga was absent among male STD clinic patients (MSPs) from the eastern province of Jiangsu (Fisher exact test $=0.036$ ), while being prevalent in the 3 southern provinces.

\section{P3.261 GLOBAL MULTILOCUS SEQUENCE TYPE (MLST) ANALYSIS OF CHLAMYDIA TRACHOMATIS STRAINS FROM 16 COUNTRIES}

doi:10.1136/sextrans-2013-051184.0717

\begin{abstract}
IJ Isaksson, ${ }^{2} \mathrm{R}$ Bom, 'I Saleh, 'B Herrmann, ${ }^{2} \mathrm{~S}$ Bruisten. 'Department of Clinical Microbiology, Uppsala, Sweden; ${ }^{2}$ Public Health Laboratory, Public Health Service of Amsterdam, Amsterdam, The Netherlands
\end{abstract}

Background The Chlamydia trachomatis MLST database was established in 2007 and is based on five target regions (non-housekeeping genes) and the conventional ompA gene. It enables worldwide epidemiological analyses.

Methods Samples were included from 11 studies focusing on specific risk groups and with different study objectives. Geographical distribution of MLST profiles was carried out and eBURST analysis identified genetic founders.

Results A total of 414 MLST profiles were recognised from 2087 entries. Polymorphism of target regions was reflected in varying numbers of alleles; $h c t B 89$, CT058 51, CT144 30, CT172 41, рыр $B 35$. With addition of 49 ompA gene variants 459 profiles exist.

There were 16 countries from which samples originated, the majority coming from the Netherlands 39\%, Sweden $16 \%$ and Norway $12 \%$, but also samples from countries in North and South America, Africa and other European countries. Overall 605 (29\%) of the database samples originated from men having sex with men (MSM) while the remaining samples were assumed to be from heterosexuals.

Singletons or small clusters emerged from 377 of the MLST profiles that were found 1-9 times while 31 profiles were found 10-43 times each. There were 8 profiles that predominated and were each found between 83 and 140 times and constituted large clusters that comprised 868 samples (41.6\%). Four of the predominating profiles were strongly associated with MSM, with $96-100 \%$ of the samples coming from MSM. In the other four large clusters heterosexuals comprised $>90 \%$ of the samples. eBURST analysis identified 3 of the 8 predominating profiles as founders, and another 3 as subgroup founders. The genetic diversity was much lower in the MSM clusters compared to clusters in heterosexuals.

Conclusions Worldwide a few C. trachomatis MLST profiles predominate. Different MLST profiles predominate among MSM and heterosexuals.

\section{P3.262 COMPARISON OF URINE SAMPLES AND PENILE SWABS IN THE DETECTION OF HUMAN PAPILLOMA VIRUS IN MEN USING THE SPF $_{10}$ LINE PROBE ASSAY}

doi:10.1136/sextrans-2013-051184.0718
'F M P A Koene, 'P F G Wolffs, 'A A T P Brink, ${ }^{2}$ N H T M Dukers-Muijrers, ${ }^{3}$ W G V Quint, ' $\mathrm{C}$ A Bruggeman, 1,2 C J P A Hoebe. 'Department of Medical Microbiology, School of Public Health and Primary Care (CAPHRI), Maastricht University Medical Center, Maastricht, The Netherlands; '2Department of Sexual Health, Infectious Diseases and Environment, South Limburg Public Health Service, Geleen, The Netherlands, ${ }^{3} D D L$ Diagnostic Laboratory, Rijswijk, The Netherlands

Background Penile swab sampling is the gold standard when testing for human papilloma virus (HPV) in men. Urine could provide a more convenient sampling material. Therefore we compared the detection and typing of HPV in urine samples and penile swabs using a highly sensitive method.

Methods First void urine and self-obtained penile swabs were collected from 120 men visiting a Sexually Transmitted Infections (STI) clinic in South Limburg, The Netherlands. When exclusion criteria were met, the results of 111 men (mean age 29.4 years) were analysed. Broad-spectrum HPV DNA amplification and genotyping were performed using $\mathrm{SPF}_{10}$-DEIA-LiPA 25 system (Labo Bio-Medical Products, Rijswijk, The Netherlands). There are 12 high risk HPV types (hrHPV), 5 possible hrHPV types and 8 low risk HPV types (lrHPV) detectable by $\mathrm{SPF}_{10} \mathrm{LiPA}_{25}$

Results HPV DNA was found in 75 (67.6\%) men. In 73 men HPV DNA was detected in the penile swab or both samples and in two men HPV DNA was detected only in the urine sample. Sixty-six paired samples were concordant in being HPV positive $(n=30)$ or negative $(n=36)$. Eleven of the 30 matching samples were concordant in their genotypes, fourteen pairs were comparable ( $\geq 1$ genotype identical) and five pairs were discordant in genotypes. Three of these discordant and one comparable urine sample contained (possible) high risk types which were not found in the swab. Furthermore, the two positive urines with a negative swab contained (possible) high risk types as well.

Conclusion Urine samples are not comparable to penile swabs in the detection of HPV in men. However, in the urine sample of six men high risk types were found that could not be detected in the penile swab alone. This could be an indication of an additional value of the urine sample aside the penile swab in the detection of HPV in men.

\section{P3.263 DO MEN HARBOUR THE SAME STRAIN OF GONOCOCCUS AT MULTIPLE ANATOMICAL SITES?}

doi:10.1136/sextrans-2013-051184.0719

K Eastick. Scottish Bacterial STI Reference Laboratory, Royal Infirmary of Edinburgh, Edinburgh, UK

Background All N. gonorrhoeae clinical isolates in Scotland are routinely typed by Neisseria gonorrhoeae multi-antigen sequence typing (NG-MAST). The frequency with which individuals harboured the same $N$. gonorrhoeae strain at $>1$ anatomical site concurrently was examined.

Methods Episodes of gonorrhoea between 2004 and January 2013 comprising typed isolates from two or more anatomical sites of the same male individual submitted within a one-month period were analysed for similarity.

Results 410 episodes of gonorrhoea were identified with $>1$ site cultured. The table shows differences in sequence type (ST) within episodes. Forty episodes with three cultures are included in all three pairwise combinations. Overall $91.2 \%$ of episodes had identical STs at all sites.

Where STs differed at only one allele, the sequences were compared using CLUSTALW. Thirteen of 14 alleles compared were at the por $B$ locus. Nine pairs were $99.8 \%$ similar, representing a difference of 1 nucleotide. The remaining pairs showed $88.4 \%, 98.2 \%$, $97.8 \%, 96.9 \%$ and $75.1 \%(t b p B)$ similarity. 
Conclusion A large majority of patients harbour the same ST of $N$. gonorrhoeae at all sites cultured. In a further $2.2 \%$ of patients there is minimal variation, which would be consistent with mutation of the por $B$ gene during the course of infection. This uniformity is not necessarily due to infection from the same partner, as some STs circulate widely.

This data adds to the understanding of the ecology of $N$. gonorrhoeae in an era where patients positive by nucleic acid amplification tests often receive limited culture for typing and susceptibility testing and assumptions may be made about the strain infecting uncultured sites. This data adds to knowledge of the frequency of mutation of the porB locus in vivo and the frequency of concurrent gonococcal infections with different strains.

\section{Abstract P3.263 Table 1}

\begin{tabular}{llll}
\hline Table 1 & & & \\
\hline Paired isolates & Identical ST & ST differing at 1 allele & ST differing at 2 alleles \\
Urethral/rectal & $156(89.6 \%)$ & $6(3.4 \%)$ & $12(6.9 \%)$ \\
Urethral/pharyngeal & $186(92.5 \%)$ & $4(2 \%)$ & $11(5.5 \%)$ \\
Rectal/pharyngeal & $104(90.4 \%)$ & $6(5.2 \%)$ & $5(4.3 \%)$ \\
\hline
\end{tabular}

\section{P3.264 GENETIC DIVERSITY OF HUMAN IMMUNODEFICIENCY VIRUS TYPE 1 IN DEMOCRATIC REPUBLIC OF CONGO: A REVIEW OF AVAILABLE DATA}

doi:10.1136/sextrans-2013-051184.0720

${ }^{1}$ E N Kamangu, ${ }^{1} Z$ Kabututu, ' $\mathrm{G}$ L Mvumbi, ${ }^{1} \mathrm{R}$ L Kalala, ${ }^{2} \mathrm{G}$ K Mesia. ${ }^{1}$ Faculty of Medicine, Department of Basic Sciences, University of Kinshasa (UNIKIN), Kinshasa, Congo; 'Faculty of Pharmaceutical Sciences and Medicine, Department of Clinical Pharmacology, University of Kinshasa (UNIKIN), Kinshasa, Congo

HIV has a genetic diversity that is equal to the complexity of its follow up of the patients. The classification of the different variants has allowed us to understand the virus, the geographical distribution and evolution of the pandemic and to better guide the follow up and the care of patients infected by HIV. Review the specifics of the HIV epidemic in the Democratic Republic of Congo (DRC), in terms of different molecular variants of HIV compared to the published location for the country. The search of the literature and abstracts presented at conferences with the subject of interest to identify different variants of HIV type 1 in the DRC on the websites of research. Online search was based on the following key words: "HIV subtype, DRC", "genotype, HIV, DRC" and "HIV strains in the Democratic Republic of Congo". It was restricted to the published literatures and presented abstracts between 1997 and 2012. According to manuscripts published since 1997, we have noticed a dominating prevalence of group $\mathrm{M}(100 \%)$ and of subtype $\mathrm{A}$ at $50.40 \%$ [31.2-68.9] for the entire country. In the Eastern part, variants A $(44.73 \%)$ are dominant on variants C $(12.20 \%), G(11.5 \%), D(9.12 \%)$ and $U(7.24 \%)$. In the Center, variants A $(62.57 \%)$ are followed by variants C $(10.32 \%), \mathrm{H}$ (5.02\%), $\mathrm{U}(4.3 \%)$ and $\mathrm{D}(3.9 \%)$. In the Western part, variants $\mathrm{A}$ $(40.91 \%)$ are followed by variants G $(19.29 \%), \mathrm{D}(10.5 \%), \mathrm{F}$ $(5.65 \%)$ and $C(4.51 \%)$. For the entire country, variants are found in the following order: A (49.40\%), G (10.73\%), C (9.01\%) and $\mathrm{D}(7.86 \%)$. The differences between and within groups are statistically significant for each variants. Several variants of HIV type 1 circulates throughout the DRC. The high number of recombinant forms (CRFs) shows the diversity and dynamics of the virus in this country.

\section{P3.265 DISCORDANT COUPLES IN HIV/AIDS CYCLE}

doi:10.1136/sextrans-2013-051184.0721

R N Mbugua. Kenya Medical Researach Institute, Nairobi, Kenya

Objectives A large proportion of new HIV infections in sub-Saharan Africa occur in stable HIV-discordant partnerships. In some couples, the strong desire to conceive a child may lead to risky behaviour despite knowledge of discordant serostatus. Our objective was to compare HIV transmission between discordant couples who did and did not conceive during participation in a clinical trial. Methods: Five hundred and thirty-two HIV-discordant couples were followed for up to 2 years in Kenya Network of Women Living with HIV/AIDS Kenya as part of the Partners in Prevention HSV/ HIV Transmission Study. Quarterly HIV-1 antibody and urine pregnancy test results were analysed. Results: Forty-one HIV-1 seroconversions occurred over 888 person-years of follow-up, resulting in an annual incidence of 4.6/100 person-years. Twenty seroconversions occurred among 186 HIV-1-uninfected individuals in partnerships in which pregnancy occurred (10.8\% of HIV-1-negative partners in this group seroconverted), in comparison to 21 seroconversions among 353 uninfected individuals in partnerships in which pregnancy did not occur (5.9\% of HIV-1-negative partners seroconverted), resulting in a relative risk of 1.8 [95\% confidence interval (CI) 1.01-3.26.

Conclusions Pregnancy was associated with an increased risk of HIV sero conversion in discordant couples. These data suggest that the intention to conceive among HIV discordant couples may be contributing to the epidemic. There are an estimated 33 million people in the world infected with HIV, $60 \%$ of whom reside in subSaharan Africa. Emerging data indicate that a large proportion of new infections in this region occur in stable HIV discordant relationships. Prevention efforts in this population have focused on couples-based HIV testing to equip partners with knowledge of their serostatus in order to motivate behaviour change.

\section{P3.266 THE PREVALENCE OF HPV GENOTYPES IN PATIENTS WITH GENITAL WARTS IN SINGAPORE - WILL THE HPV VACCINE BE USEFUL IN THIS POPULATION?}

doi:10.1136/sextrans-2013-051184.0722

${ }^{1} \mathbf{Y}$ Yew, ' $\mathrm{P}$ Sen, ${ }^{\mathrm{T}} \mathrm{T}$ Chio, ${ }^{2} \mathrm{E}$ Koay, ${ }^{1} \mathrm{R}$ Chan. ' National Skin Centre, Singapore, Singapore; ${ }^{2}$ National University Health System, Singapore, Singapore

Introduction Worldwide, $90 \%$ of genital warts are caused by HPV types 6 and 11. A HPV vaccine covering HPV 6 and 11 is now available. To evaluate its potential benefits, we aim to characterise the prevalence of the HPV genotypes in genital warts in Singapore.

Methods We utilised a validated commercialised genotyping assay, the HybriBio HPV GenoArray test that is able to identify 21 HPV types including 5 low-risk types $(6,11,42,43$, and 44). After a prior pilot study of ten patients, a total of 100 patients with genital warts and no prior treatment were recruited into this study. Scrapings from the warts were performed, stored in virus transport medium and DNA was then extracted for analysis. Demographics, sexual history and clinical findings were collected using a self-administered questionnaire.

Results There were 71 male and 29 female patients. The average age of the patients was 32.1 years. The majority (49\%) were single and heterosexual. Approximately $50 \%$ of the patients had an average of more than five lifetime sexual partners. The majority (69\%) had genital warts for the first time. HPV genotypes were characterised in $92 \%$ of the patients. Either HPV 6 and/or HPV 11 was detected in $87.0 \%$ of the patients. Thirty-four patients had high-risk HPV genotypes detected in their genital warts.

Conclusion A simple scraping methodology from genital warts followed by HPV typing (HybriBio HPV GenoArray test) has been shown 